\title{
Calcium Pyrophosphate Dihydrate (CPPD) Crystal Deposition Disease, Crowned Dens Syndrome (CDS) and Its Mimicries: Case Report
}

\section{Symrenjit Surjit Singh ${ }^{1 *} \mid$ Hui Jean Lee ${ }^{1,2,3}$}

*Correspondence: Symrenjit Surjit Singh

Address: ${ }^{1}$ Greenslopes Private Hospital, Queensland, Australia; ${ }^{2}$ Princess Alexandra Hospital, Queensland, Australia; ${ }^{3}$ University of Queensland, Queensland, Australia

e-mail $\bowtie$ : symrensaini@gmail.com

Received: 17 November 2021; Accepted: 22 November 2021

Copyright: (C) 2021 Surjit Singh S. This is an open-access article distributed under the terms of the Creative Commons Attribution License, which permits unrestricted use, distribution, and reproduction in any medium, provided that the original work is properly cited.

\section{ABSTRACT}

Headache is one of the most encountered presentations worldwide. Hence, the approach in which practitioners take in investigating, diagnosing and managing it is crucial given the high complication rates from secondary causes seen more commonly in the elderly population. We discuss a case of a patient with chronic headaches, features of occipital neuralgia and neck pain who was eventually diagnosed with Crowned Dens Syndrome (CDS). We intend to highlight this unique presentation of Calcium Pyrophosphate crystal deposition (CPPD) disease in the spine and the need to consider a broad range of differentials especially in an elderly individual presenting with headaches.

Keywords: Acute Headaches, Headaches in Elderly, Calcium Pyrophosphate Dihydrate Crystal Deposition, Crowned Dens Syndrome, Cervical Arthropathy

\section{Introduction}

An elderly lady presented to her geriatrician with chronic headaches, occipital neuralgia and neck pain. Headache is a relatively common presentation and it is key to rule out common secondary causes (infection, cerebrovascular accidents, rheumatological and malignancies) before considering rarer differentials such as CDS (Kaniecki and Levin, 2019). CDS involves the deposition of CPPD crystals around the odontoid process which leads to inflammation within the joint (Bouvet et al., 1985).

Case

An 88-year-old lady presented with chronic headaches. Her co-morbidities include short term memory deficits, systolic hypertension, aortic regurgitation, osteoarthritis and recurrent syncopal episodes secondary to orthostatic hypotension. Over the last 2-3 weeks, she noticed a worsening of her chronic headaches and reported sharp throbbing pains which began in the occipital region. It radiated to the frontal region and was also accompanied by neck pain. She denied pain radiating to the arm, sensory 
or motor deficits. Aggravating factors included activities which require a forward flexion of the neck (e.g., reading and knitting). She reported no other neurological or constitutional symptoms (fever, loss of weight or a loss of appetite). There was also no trauma to the surrounding region, history of malignancy or infections. She denied joint stiffness, muscle weakness or a history of inflammatory arthritis.

On physical examination, she was alert and orientated (Glasgow Coma Scale of 15) with a normal inspection of the cervical spine region. Palpation of the cervical paravertebral region elicited tenderness, especially around the C2-C5 region. A normal cervical spine range of motion was established with occasional crepitus and no pain on movement. A Spurling's test was negative and no neurological deficits (motor or sensory) were identified including negative Kernig and Brudzinski signs.

Laboratory investigations were almost normal with an Erythrocyte Sedimentation Rate (ESR) of 12 $\mathrm{mm} / \mathrm{h}$ and a borderline elevated C-Reactive Protein (CRP) of $5 \mathrm{mg} / \mathrm{dL}$. The initial imaging of choice included a Magnetic Resonance Imaging (MRI) scan and carotid ultrasonography, both of which were reported as normal. However, a Computed Tomography (CT) scan of her spine demonstrated calcification of the periodontal transverse ligament, posterior longitudinal ligament and ligamentum flavum at the region of C2/C3 and C3/C4 consistent with CPPD disease. There were minor degenerative changes and osteophytes reported in the C4-C7 region with narrowing of the left C6 neural foramen causing impingement (Fig. 1 and Fig. 2).

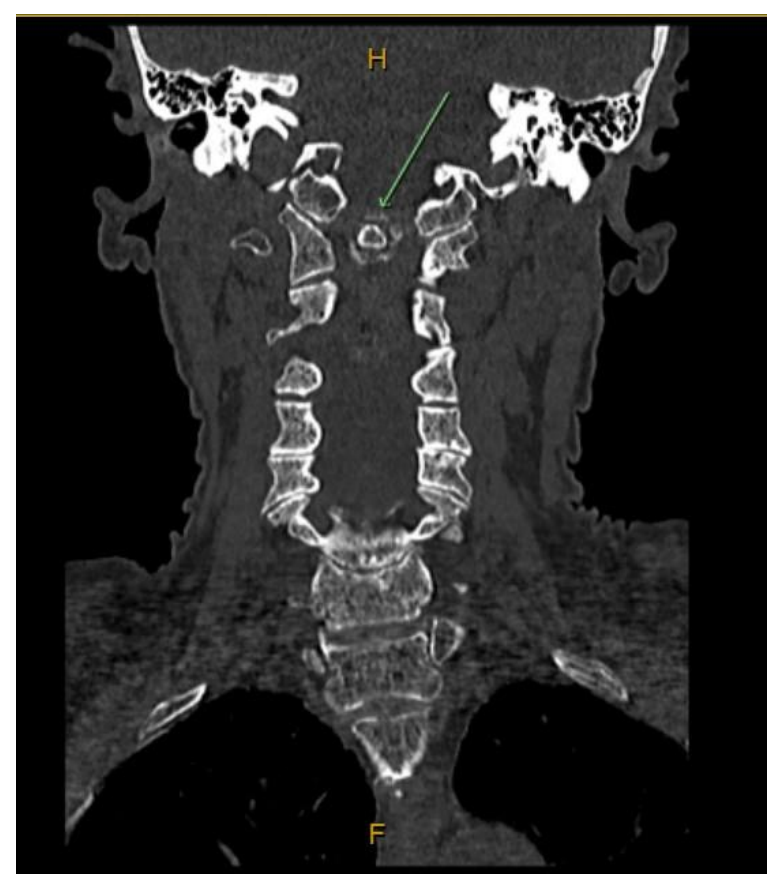

Figure 1: Non-contrast Coronal CT demonstrating CPPD deposition of the ligaments in a circular or crowned appearance (green arrow) consistent with CDS. 


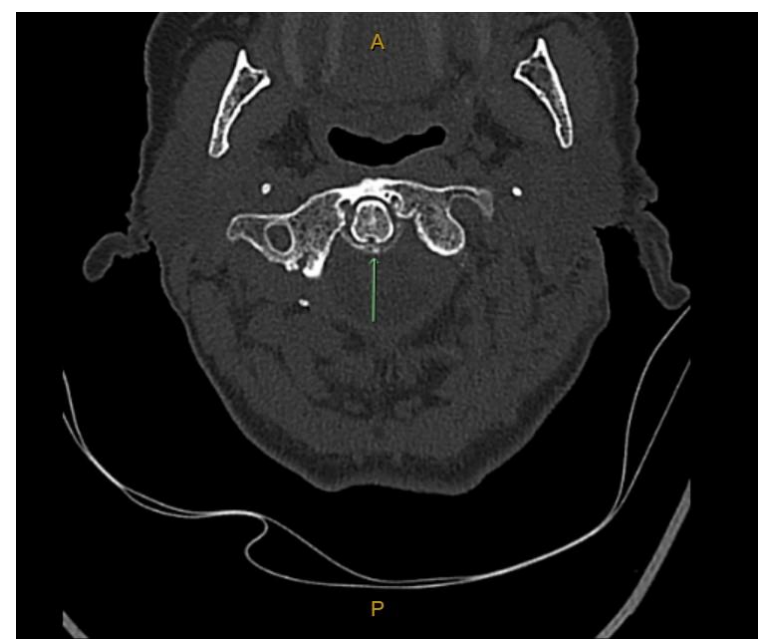

Figure 2: Axial CT showing transverse ligament calcification (green arrow) consistent with CPPD disease.

Management included pain relief (Nonsteroidal anti-inflammatory drugs (NSAIDs) and Paracetamol) and heat packs. Thereafter, she reported good improvement of her symptoms with a reduction in pain scores and an improved quality of sleep. She was then referred on to the physiotherapist for commencement of neck mobility exercises.

\section{Discussion}

Headache is a common complaint seen in any healthcare setting. When approaching a presentation of such, especially acutely, it is important to rule out red flags such as infections (meningitis, epidural abscesses and osteomyelitis), cerebrovascular accidents, rheumatological causes (giant cell arteritis or polymyalgia rheumatica), primary musculoskeletal degenerative conditions or worse yet malignancies (primary or metastatic) before making a diagnosis of primary headaches (Kaniecki and Levin, 2019). Despite the fact that primary headaches remain $2 / 3$ of the cause, elderly individuals are 12 times more likely to have serious underlying pathologies which may present differently as compared to the younger population (Kaniecki and Levin, 2019). Other less common differentials, such as crystal deposition, should be considered once life-threatening causes have been ruled out.

Crystal arthropathy of the spine is less commonly heard of as deposition is mostly seen in the peripheries. The reason behind its deposition in the spine is still not well established and has been attributed to tissue changes such as necrosis, injuries or degeneration (Hsu et al., 2002). Additionally, de novo deposition is relatively common in individuals $>65$ years old (Mikhael et al., 2013). Examples of crystal arthropathies of the joint and soft tissue include gout which is caused by monosodium urate (MSU), CPPD, calcium oxalate and basic calcium phosphate (Rubenstein and Pritzker, 1989). Gout is said to more commonly affect the lumbar spine while CPPD deposits in the thoracic region. Presentations of both could vary from minimal (cervical myelopathy and radiculopathy) to severe (spinal stenosis and 
cauda equina syndrome) (Lam et al., 2007). To date, there have been no reports of basic calcium phosphate deposition in the spine. Ideally, the diagnosis of spinal gout is made by identifying negatively birefringent MSU crystals on fine needle aspiration cytology or biopsy as imaging findings may be nonspecific and could mimic infections and metastasis (Jegapragasan et al., 2014; Chan et al., 2009) There has been emerging evidence on the use of Dual Energy Computed Tomography (DECT) scans in diagnosing gout peripherally; however, there is no validated data regarding its use in regions such as the shoulders, hip, spine and pelvis (Chou et al., 2017).

Several case reports of gout and pseudogout in the spine have demonstrated clinical (acute onset of fever, back pain and neurological deficits), laboratory (elevated inflammatory markers) and radiological findings which closely mimic infections such as pyogenic spondylitis (Mikhael et al., 2013, Bridges et al., 2017). Although osteomyelitis typically presents acutely, it is important to be mindful of chronic and tuberculous osteomyelitis which is slow and indolent (Yoshikawa and Cunha, 2002).

First described by Bouvet. et al. in 1985, CDS, as the name implies, is a crowned shaped deposition of CPPD crystals around the odontoid process (ligaments, synovial membrane and articular capsule) causing inflammation within the joint (Bouvet et al., 1985; Goto et al., 2007). A retrospective study looking into incidental calcium deposition on CT scans showed that odontoid calcifications were present in $15.9 \%$ of patients. However, only $12.5 \%$ were symptomatic (Sano et al., 2018).

CPPD arthropathy is commonly seen in patients of increasing age, prior injuries and surgeries. It is also associated with osteoarthritis, chronic kidney disease and endocrine disorders (haemochromatosis, hypomagnesemia, hyperparathyroidism, hypophosphatemia, Wilson's disease and familial hypocalciuric hypercalcemia) (Rosenthal, 2020). The above mentioned should be screened for in patients aged $<60$ years who present with florid polyarticular CPPD. Although there are hereditary predispositions to CPPD arthropathy, the most common is the sporadic form (Rosenthal and Ryan, 2016).

In acute CPPD arthritis, patients typically present with mono- or oligoarthritis, inflammatory and constitutional symptoms mimicking gout or septic arthritis. It commonly affects the lower (knee) and upper limbs (wrist). However, axial manifestations involving the temporomandibular joint and spine (intervertebral disc, facet joints, neural foramen and ligaments) are possible (Rosenthal, 2020, Naqvi et al., 2008).

Chronic CPPD arthritis, which is more common, presents with chronic polyarticular joint involvement resembling osteoarthritis, rheumatoid arthritis and polymyalgia rheumatica (Rosenthal, 2020). With that being said, both osteo- and CPPD arthritis are common in older patients and have similar 
risk factors (e.g., increasing age and previous injuries) (Zhang et al., 2011). Studies have shown that $1 / 3$ of patients with osteoarthritis undergoing knee arthroscopy had CPPD deposition (Macmullan and McCarthy, 2012). Although there have been associations described, it is of no surprise that it could cooccur by chance (Rosenthal, 2020).

A diagnosis of CPPD arthropathy is made by identifying positively birefringent crystals in synovial fluid analysis and/or the presence of radiographic evidence (Rosenthal, 2020). In CDS, an unenhanced CT of the cervical region is the preferred modality of imaging. It demonstrates CPPD deposition in a 'crownlike' shape surrounding the dens apex or ligaments (transverse or flavum) (Rosenthal, 2020). As reported, there is still a limited use for DECT in diagnosing CPPD disease (sensitivity of 55\% and specificity of 92\%). Other modalities reported a (sensitivity and specificity) as follows: ultrasound (91\% and 92\%), conventional radiography (36\% and 90\%) and suspected clinical diagnosis (82\% and 88\%) (Kravchenko et al., 2021). MRI is usually not helpful in assessing CPPD deposition, as seen in the case above, due to the absence of signals from the calcifications (Rosenthal and Ryan, 2016).

Management of CPPD arthropathy includes the use of NSAIDs which is said to provide relatively quick pain relief. Other treatment options include colchicine, corticosteroids (i.e., intra-articular or systemic) or even combinations of the two (Rosenthal and Ryan, 2016). Although there have been trials, evidence is still lacking surrounding the use of disease modifying agents (Rosenthal and Ryan, 2016). CDS generally carries a good prognosis, although physicians should be mindful of the rare neurological complications (e.g., myelopathy of the cervical spine and spinal stenosis) due to large crystal depositions (Goto et al., 2007).

\section{Conclusion}

CPPD disease, as described, has multiple clinical mimicries and associations hence posing a challenge of diagnosis. We are therefore hoping to increase awareness of this medical phenomenon amongst the elderly population hence reducing the need for unnecessary interventions (e.g., imaging and antimicrobial use).

\section{References}

Bouvet JP, Parc JM, Michalski B, Benlahrache C, Auquier L. Acute neck pain due to calcifications surrounding the odontoid process: the crowned dens syndrome. Arthritis Rheum 1985; 28: 1417-1420.

Bouvet JP, Parc JM, Michalski B, Benlahrache C, Auquier L. Pseudogout of the cervical and thoracic spine mimicking infection after lumbar fusion: case report. J Neurosurg Spine 2017; 27: 145-149.

Chan AT, Leung JL, Sy AN, Wong WW, Lau KY, Ngai WT, Tang VW. Thoracic spinal gout mimicking metastasis. Hong Kong 
Med J 2009; 15: 143-145.

Chou H, Chin TY, Peh WC. Dual-energy CT in gout - A review of current concepts and applications. J Med Radiat Sci 2017; 64: 41-51.

Goto S, Umehara J, Aizawa T, Kokubun S. Crowned Dens syndrome. J Bone Joint Surg Am 2007; 89: 2732-2736.

Hsu CY, Shih TT, Huang KM, Chen PQ, Sheu JJ, Li YW. Tophaceous gout of the spine: MR imaging features. Clin Radiol 2002; 57: 919-925.

Jegapragasan M, Calniquer A, Hwang WD, Nguyen QT, Child Z. A case of tophaceous gout in the lumbar spine: a review of the literature and treatment recommendations. Evid Based Spine Care J 2014; 5: 52-56.

Kaniecki RG and Levin AD. Headache in the elderly. Handb Clin Neurol 2019; 167: 511-528.

Kravchenko D, Karakostas P, Kuetting D, Meyer C, Brossart P, Behning C, Schäfer VS. The role of dual energy computed tomography in the differentiation of acute gout flares and acute calcium pyrophosphate crystal arthritis. Clin Rheumatol 2021; 9: 1-1.

Lam HY, Cheung KY, Law SW, Fung KY. Crystal arthropathy of the lumbar spine: a report of 4 cases. J Orthop Surg (Hong Kong) 2007; 15: 94-101.

MacMullan $\mathrm{P}$ and McCarthy G. Treatment and management of pseudogout: insights for the clinician. Ther Adv Musculoskelet Dis 2012; 4: 121-131.

Mikhael MM, Chioffe MA, Shapiro GS. Calcium pyrophosphate dihydrate crystal deposition disease (pseudogout) of lumbar spine mimicking osteomyelitis-discitis with epidural phlegmon. Am J Orthop (Belle Mead NJ) 2013; 42 : E64-E67.

Naqvi AH, Abraham JL, Kellman RM, Khurana KK. Calcium pyrophosphate dihydrate deposition disease (CPPD)/Pseudogout of the temporomandibular joint - FNA findings and microanalysis. Cytojournal 2008; 5: 8.

Rosenthal AK and Ryan LM. Calcium Pyrophosphate Deposition Disease. N Engl J Med 2016; 374: 2575-2584.

Rosenthal AK. Clinical manifestations and diagnosis of calcium pyrophosphate crystal deposition (CPPD) disease. In: DALBETH, N. \& ROMAIN, P. (eds.) UptoDate. Wellesley, Massachusetts, 2020.

Rubenstein J and Pritzker KP. Crystal-associated arthropathies. AJR Am J Roentgenol 1986; 152: 685-695.

Sano M, Yamashita S, Aiba T. The prevalence of calcification around odontoid process and the incidence of crowned dens syndrome in the neurosurgical ward: A single institution's analysis. Mod Rheumatol 2018; 28: 182-187.

Yoshikawa TT and Cunha BA. Osteomyelitis in Elderly Patients. Clin Infect Dis 2002; 35: 287-293.

Zhang W, Doherty M, Bardin T, Barskova V, Guerne PA, Jansen TL, Leeb BF, Perez-Ruiz F, Pimentao J, Punzi L, Richette P. European League Against Rheumatism recommendations for calcium pyrophosphate deposition. Part I: terminology and diagnosis. Ann Rheum Dis 2011; 70: 563-570. 